Rico Berner, Jakub Sawicki, Eckehard Schöll

\title{
Birth and Stabilization of Phase Clusters by Multiplexing of Adaptive Networks
}

Journal article | Accepted manuscript (Postprint)

This version is available at https://doi.org/10.14279/depositonce-10858

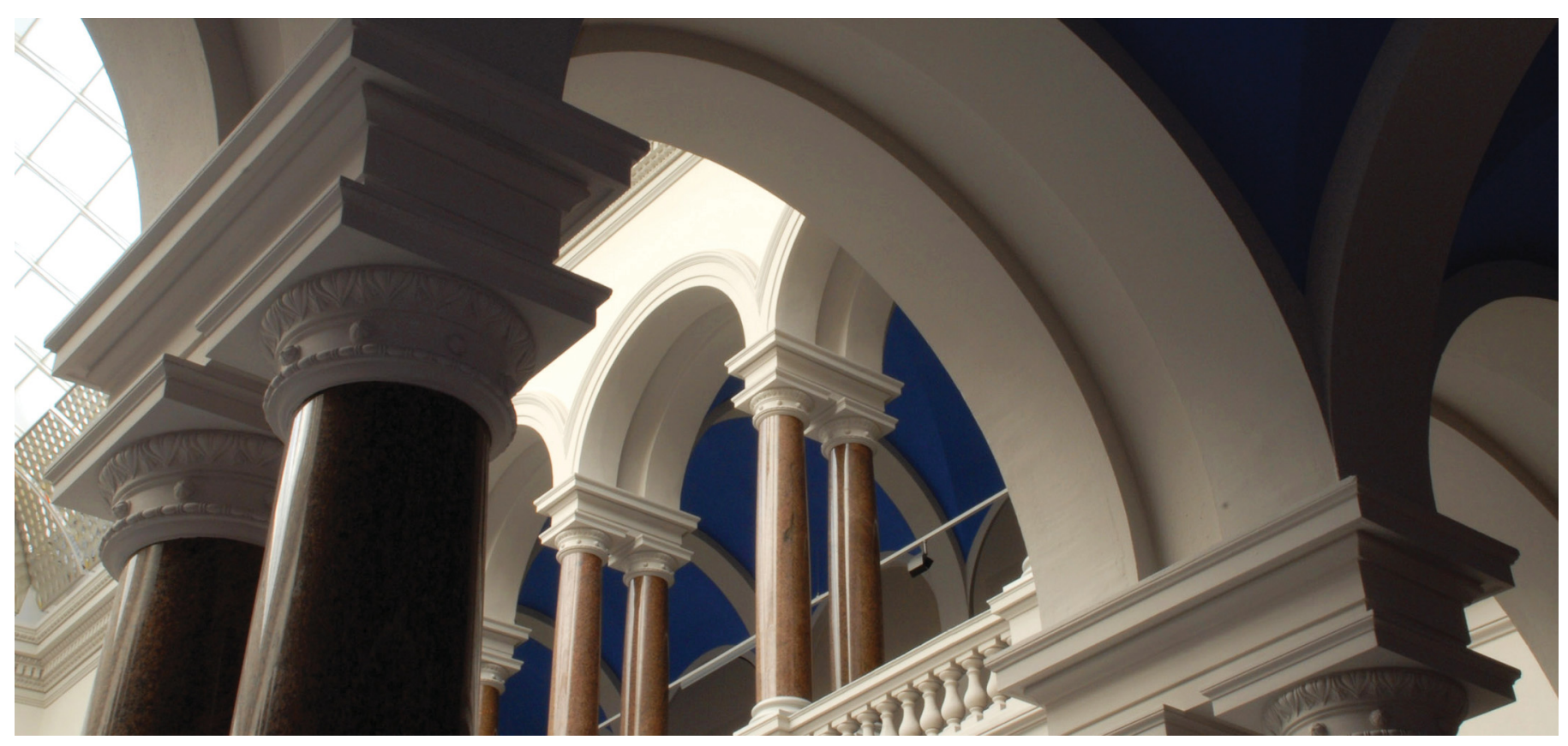

Berner, R., Sawicki, J., \& Schöll, E. (2020). Birth and Stabilization of Phase Clusters by Multiplexing of Adaptive Networks. Physical Review Letters, 124(8). https://doi.org/10.1103/physrevlett.124.088301 (c) 2020 American Physical Society 


\title{
Birth and stabilization of phase clusters by multiplexing of adaptive networks
}

\author{
Rico Berner ${ }^{1,2},{ }^{*}$ Jakub Sawicki ${ }^{1}$, and Eckehard Schöll ${ }^{1 \dagger}$ \\ ${ }^{1}$ Institut für Theoretische Physik, Technische Universität Berlin, Hardenbergstr. 36, 10623 Berlin, Germany and \\ ${ }^{2}$ Institut für Mathematik, Technische Universität Berlin, Hardenbergstr. 36, 10623 Berlin, Germany
}

(Dated: January 14, 2020)

\begin{abstract}
We propose a concept to generate and stabilize diverse partial synchronization patterns (phase clusters) in adaptive networks which are widespread in neuro- and social sciences, as well as biology, engineering, and other disciplines. We show by theoretical analysis and computer simulations that multiplexing in a multilayer network with symmetry can induce various stable phase cluster states in a situation where they are not stable or do not even exist in the single layer. Further, we develop a method for the analysis of Laplacian matrices of multiplex networks which allows for insight into the spectral structure of these networks enabling a reduction to the stability problem of single layers. We employ the multiplex decomposition to provide analytic results for the stability of the multilayer patterns. As local dynamics we use the paradigmatic Kuramoto phase oscillator, which is a simple generic model and has been successfully applied in the modeling of synchronization phenomena in a wide range of natural and technological systems.
\end{abstract}

Complex networks are an ubiquitous paradigm in nature and technology, with a wide field of applications ranging from physics, chemistry, biology, neuroscience, to engineering and socio-economic systems. Of particular interest are adaptive networks, where the connectivity changes in time, for instance, the synaptic connections between neurons are adapted depending on the relative timing of neuronal spiking [1-5]. Similarly, chemical systems have been reported [6], where the reaction rates adapt dynamically depending on the variables of the system. Activity-dependent plasticity is also common in epidemics [7] and in biological or social systems [8]. Synchronization is an important feature of the dynamics in networks of coupled nonlinear oscillators [9-13]. Various synchronization patterns are known, like cluster synchronization where the network splits into groups of synchronous elements [14], or partial synchronization patterns like chimera states where the system splits into coexisting domains of coherent (synchronized) and incoherent (desynchronized) states [15-17]. These patterns were also explored in adaptive networks [18-33]. Furthermore, adapting the network topology has also successfully been used to control cluster synchronization in delay-coupled networks [34].

Another focus of recent research in network science are multilayer networks, which are systems interconnected through different types of links [35-38]. A prominent example are social networks which can be described as groups of people with different patterns of contacts or interactions between them [39-41]. Other applications are communication, supply, and transportation networks, for instance power grids, subway networks, or airtraffic networks [42]. In neuroscience, multilayer networks represent for instance neurons in different areas of the brain, neurons connected either by a chemical link or by an electrical synapsis, or the modular connectivity structure of brain regions [43-51]. A special case of multilayer networks are multiplex topologies, where each layer contains the same set of nodes, and only pairwise connections between corresponding nodes from neighbouring layers exist $[52-71]$.

In spite of the lively interest in the topic of adaptive networks, little is known about the interplay of adaptively coupled groups of networks [25, 72, 73]. Such adaptive multilayer or multiplex networks appear naturally in neuronal networks, e.g., in interacting neuron populations with plastic synapses but different plasticity rules within each population $[74,75]$, or affected by different mechanisms of plasticity [76], or the transport of metabolic resources [77]. Beyond brain networks, coexisting forms of (meta)plasticity are investigated in neuroinspired devices to develop artificially intelligent learning circuitry [78].

In this Letter we show that a plethora of novel patterns can be generated by multiplexing adaptive networks. In particular, partial synchronization patterns like phase clusters and more complex cluster states which are unstable in the corresponding monoplex network can be stabilized, or even states which do not exist in the single-layer case for the parameters chosen, can be born by multiplexing. Thus our aim is to provide fundamental insight into the combined action of adaptivity and multiplex topologies. Hereby we elucidate the delicate balance of adaptation and multiplexing which is a feature of many real-world networks even beyond neuroscience [79-82]. As local dynamics we use the paradigmatic Kuramoto phase oscillator model, which is a simple generic model and has been successfully applied in the modeling of synchronization phenomena in a wide range of natural and technological systems [13].

A general multiplex network with $L$ layers each consisting of $N$ identical adaptively coupled phase oscillators 
is described by

$$
\begin{aligned}
\dot{\phi}_{i}^{\mu}= & \omega-\frac{1}{N} \sum_{j=1}^{N} \kappa_{i j}^{\mu} \sin \left(\phi_{i}^{\mu}-\phi_{j}^{\mu}+\alpha^{\mu \mu}\right) \\
& -\sum_{\nu=1, \nu \neq \mu}^{L} \sigma^{\mu \nu} \sin \left(\phi_{i}^{\mu}-\phi_{i}^{\nu}+\alpha^{\mu \nu}\right), \\
\dot{\kappa}_{i j}^{\mu}= & -\epsilon\left(\kappa_{i j}^{\mu}+\sin \left(\phi_{i}^{\mu}-\phi_{j}^{\mu}+\beta^{\mu}\right)\right),
\end{aligned}
$$

where $\phi_{i}^{\mu} \in[0,2 \pi)$ represents the phase of the $i^{\text {th }}$ oscillator $(i=1, \ldots, N)$ in the $\mu^{\text {th }}$ layer $(\mu=1, \ldots, L)$, and $\omega$ is the natural frequency. The interaction between the oscillators within each layer is determined adaptively by the intra-layer coupling weights $\kappa_{i j}^{\mu} \in[-1,1]$, whereas between the layers the inter-layer coupling weights $\sigma^{\mu \nu} \geq 0$ are fixed. The parameters $\alpha^{\mu \nu}$ are the phase lags of the interaction [83]. The adaptation rate $0<\epsilon \ll 1$ separates the time scales of the slow dynamics of the coupling weights and the fast dynamics of the oscillatory system. The phase lag parameter $\beta^{\mu}$ of the adaptation function $\sin \left(\phi_{i}^{\mu}-\phi_{j}^{\mu}+\beta^{\mu}\right)$, also called plasticity rule in the neuroscience terminology [18], describes different rules that may occur in neuronal networks. For instance, for $\beta^{\mu}={ }_{(+)} \pi / 2$, an (anti-) Hebbian-like rule [84-86] is obtained where the coupling $\kappa_{i j}$ increases (decreases) between any two systems with close-by phases [87]. If $\beta=0$, the link $\kappa_{i j}$ will be strengthened if the $i^{\text {th }}$ oscillator is advancing the $j^{\text {th }}$. Such a relationship is typical for spike-timing dependent plasticity in neuroscience $[3,5,88,89]$.

Let us note important properties of our model (1), which has been widely used as a paradigmatic model for adaptive networks [18-30] and generalizes the KuramotoSakaguchi model with fixed coupling topology [90-94]. First, $\omega$ can be set to zero without loss of generality due to the shift-symmetry of Eq.(1), i.e., considering the co-rotating frame $\phi \rightarrow \phi+\omega t$. Moreover, due to the existence of the attracting region $G \equiv\left\{\left(\phi_{i}^{\mu}, \kappa_{i j}^{\mu}\right): \phi_{i}^{\mu} \in(0,2 \pi],\left|\kappa_{i j}^{\mu}\right| \leq 1, i, j=1, \ldots, N\right.$, $\mu=1, \ldots, L\}$, one can restrict the range of the coupling weights to the interval $-1 \leq \kappa_{i j} \leq 1$ [23]. Finally, based on the parameter symmetries of the model

$$
\begin{aligned}
(\boldsymbol{\alpha}, \boldsymbol{\beta}, \boldsymbol{\phi}, \boldsymbol{\kappa}) & \mapsto(-\boldsymbol{\alpha}, \pi-\boldsymbol{\beta},-\boldsymbol{\phi}, \boldsymbol{\kappa}), \\
\left(\alpha^{\mu \mu}, \beta^{\mu}, \phi_{i}^{\mu}, \kappa_{i j}^{\mu}\right) & \mapsto\left(\alpha^{\mu \mu}+\pi, \beta^{\mu}+\pi, \phi_{i}^{\mu},-\kappa_{i j}^{\mu}\right),
\end{aligned}
$$

where $\boldsymbol{\alpha}, \boldsymbol{\beta}, \boldsymbol{\phi}, \boldsymbol{\kappa}$ abbreviate the whole set of variables and parameters, it is sufficient to analyze the system within the parameter region $\alpha^{11} \in[0, \pi / 2), \alpha^{\mu \mu} \in[0, \pi)(\mu \neq 1)$, $\alpha^{\mu \nu} \in[0,2 \pi)(\mu \neq \nu)$ and $\beta^{\mu} \in[-\pi, \pi)$.

Before we consider multiple layers, we suggest that each solution of Eq. (1) for $L=1,2$ is called a monoplex or duplex state, respectively. Already for a single layer, Eq. (1) possesses a huge variety of dynamical (monoplex) states such as multiclusters with respect to frequency synchronization, chaotic attractors, and chimera-like states, which have been studied numerically and analytically [18-23]. In particular, it has been shown that starting from uniformly distributed random initial condition $\phi_{i} \in[0,2 \pi), \kappa_{i j} \in[-1,1]$ the system can reach different frequency multicluster states with hierarchical structure depending on the parameters $\alpha$ and $\beta$. The frequency multiclusters in turn consist of several one-clusters which determine the existence and stability of the former [24]. Therefore, these one-cluster states (with identical frequency, but different phase distributions) constitute the building blocks of adaptively coupled phase oscillators, and their generalization to the multiplex case will be in the focus of this Letter. The reason for this focus is that one-cluster states, which are analytically very well understood, are building blocks for more complex dynamical states. Chimera-like states as they were studied in $[23,25]$ exist close to the borders of these states, so the existence and stability of one-clusters may pave the way for observing those hybrid patterns.

In general, one-cluster states are given by equilibria relative to a co-rotating frame [22]

$$
\begin{aligned}
\phi_{i}^{\mu} & =\Omega t+a_{i}^{\mu}, \\
\kappa_{i j}^{\mu} & =-\sin \left(a_{i}^{\mu}-a_{j}^{\mu}+\beta^{\mu}\right),
\end{aligned}
$$

with collective frequency $\Omega$ and relative phases $a_{i}^{\mu}$. Hence the second moment order parameter $R_{2}\left(\mathbf{a}^{\mu}\right)=$ $\frac{1}{N}\left|\sum_{j=1}^{N} e^{\mathrm{i} 2 a_{j}^{\mu}}\right|$ with $\mathbf{a}^{\mu} \equiv\left(a_{1}^{\mu}, \ldots, a_{N}^{\mu}\right)^{T}$ can be used as a characteristic measure. In the case of monoplex systems $(L=1)$, three types of solutions exist (see Fig. 1) which are characterized by corresponding frequencies $\Omega$ as a function of $\left(\alpha^{11}, \beta^{1}\right)$ [22]: (a) $\Omega=\cos \left(\alpha^{11}-\beta^{1}\right) / 2$ if $R_{2}\left(\mathbf{a}^{1}\right)=0$ (Splay state), (b) $\Omega=\sin \alpha^{11} \sin \beta^{1}$ if $R_{2}\left(\mathbf{a}^{1}\right)=1$ with $a_{i}^{1} \in\{0, \pi\}$ (Antipodal state), (c) $\Omega=\cos \left(\alpha^{11}-\beta^{1}\right) / 2-R_{2}(\mathbf{a}) \cos \left(\psi_{Q}\right) / 2$ if $0<R_{2}\left(\mathbf{a}^{1}\right)<1$ with $a_{i}^{1} \in\left\{0, \pi, \psi_{Q}, \psi_{Q}+\pi\right\}$ (Double antipodal state) with $\psi_{Q}$ being the unique solution (modulo $2 \pi$ ) of

$$
(1-q) \sin \left(\psi_{Q}-\alpha^{11}-\beta^{1}\right)=q \sin \left(\psi_{Q}+\alpha^{11}+\beta^{1}\right),
$$

where $q=Q / N$ and $Q \in\{1, \ldots, N-1\}$ denotes the number of relative phases $a_{i}^{1} \in\{0, \pi\}$. Here, splay states are defined in a more general sense by $R_{2}\left(\mathbf{a}^{1}\right)=0$, which includes the states $a_{i}^{1}=2 \pi i / N$ usually referred to as splay state [95].

Let us now consider these one-cluster states in multiplex structures. Therefore, we introduce the notion of lifted one-cluster states, where in each layer the state $\left(\phi_{i}^{\mu}(t), \kappa_{i j}^{\mu}(t)\right)$ is a monoplex one-cluster, i.e., the phases $a_{i}^{\mu}$ of the oscillators are of splay, antipodal, or double antipodal type which solves Eq. (3). It can be shown [102] that in duplex systems $(L=2)$ the phase difference of oscillators between the layers $\Delta a \equiv a_{i}^{1}-a_{i}^{2}$ takes only two values and solves $\Delta \Omega=\sigma^{12} \sin \left(\Delta a+\alpha^{12}\right)+\sigma^{21} \sin (\Delta a-$ $\left.\alpha^{21}\right)$, where $\Delta \Omega \equiv \Omega\left(\alpha^{11}, \beta^{1}\right)-\Omega\left(\alpha^{22}, \beta^{2}\right)$ is given above for the three different one-cluster states (splay, antipodal, double antipodal). Figure 2 displays lifted states of 


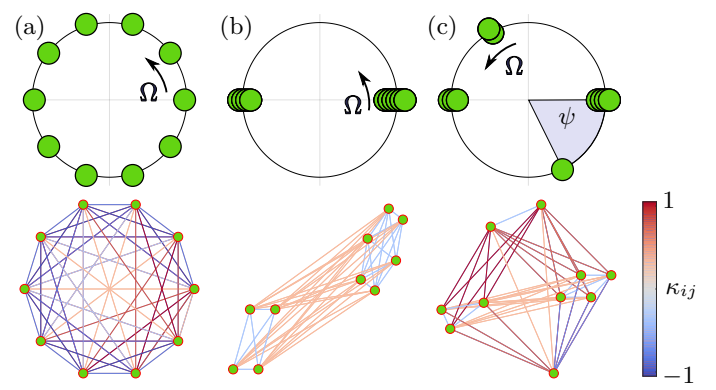

FIG. 1. Illustration of the three types of monoplex one-cluster states of Eq. (2) $(L=1)$ for an ensemble of 10 oscillators (green circles) with frequencies $\Omega$ (upper panels) and coupling structure with weights $\kappa_{i j}$ (lower panels): One-cluster (a) of splay type $\left(R_{2}(\mathbf{a})=0\right)$, (b) of antipodal type $\left(R_{2}(\mathbf{a})=1\right)$, and (c) of double antipodal type with $Q=7$. Parameters: $\alpha=0.1 \pi, \beta=0.1 \pi$

splay (a), antipodal (b), and splay type (d). The phase distributions in both layers are the same but shifted by the constant value $\Delta a$ in agreement with the above equation. In contrast to the lifted states, Fig. 2(c) shows another possible one-cluster for the duplex network. Due to the interaction of the two layers we can find a phase distribution which is of double antipodal type in each layer but not a lifted state since neither $\psi^{1}$ nor $\psi^{2}$ solve Eq. (3) for $Q=30$. This means that these states are born by the duplex set-up. Moreover, in contrast to the other examples the phase distribution between the layers does not agree, $\psi^{1} \neq \psi^{2}$. For the monoplex case, it has been shown that double antipodal states are unstable for any set of parameters [24]. Hence, finding stable double antipodal states which interact through the duplex structure is unexpected.

For more insight into the birth of phase-locked states by multiplexing, Fig. 3 displays the emergence of double antipodal states in a parameter regime where they do not exist in single-layer networks. They are characterized by the second moment order parameter $R_{2}$. It is remarkable that the new double antipodal state can be found for a wide range of the inter-layer coupling strength larger than a certain critical value $\sigma_{c}$, and is clearly different from those of the monoplex. Moreover, these states are even robust for inhomogeneous natural frequencies [102]. Below the critical value $\sigma_{c}$, the double antipodal states are no longer stable, and more complex temporal dynamics occurs which causes temporal changes in $R_{2}$. This leads to non-vanishing temporal variance indicated by the error bars in Fig. 3.

In the following we show how the dynamics in a neighborhood of theses states can be lifted as well, i.e., we investigate their local stability. The linearization of Eq. (1) around the one-cluster states described by Eq. (2) is exemplified for antipodal states but can be generalized to

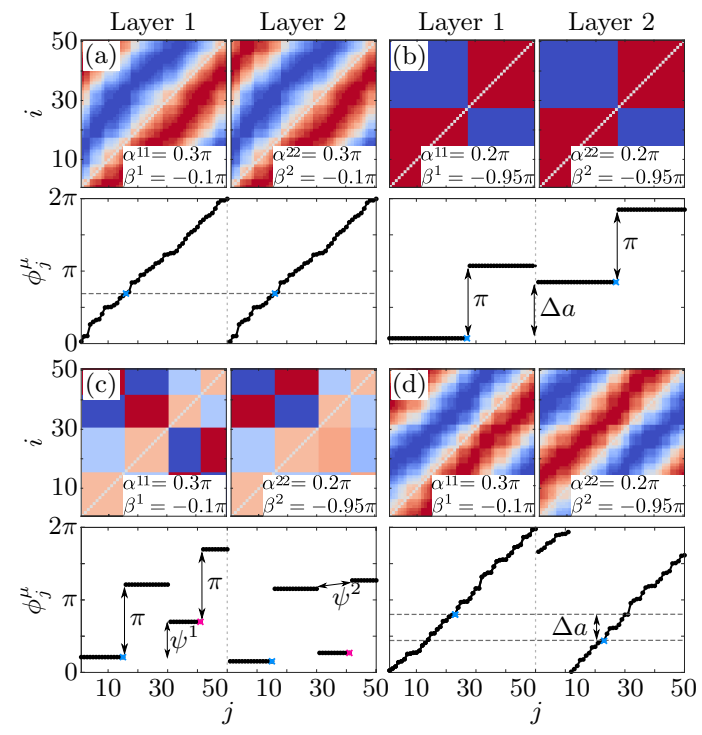

FIG. 2. Different duplex states of Eq. (2) $(L=2)$ for an ensemble of 50 oscillators in each layer with color-coded coupling weights $\kappa_{i j}^{\mu}$ (upper panels, color code as in Fig.1), phases $\phi_{j}^{\mu}$ (lower panels): Duplex one-cluster states (a) of lifted splay type $\left(R_{2}\left(\mathbf{a}^{\mu}\right)=0\right)$ for $\alpha^{12 / 21}=0.3 \pi, \sigma^{12 / 21}=0.07$; (b) of lifted antipodal type $\left(R_{2}\left(\mathbf{a}^{\mu}\right)=1\right)$ for $\alpha^{12}=0.3 \pi$, $\alpha^{21}=0.75 \pi, \sigma^{12 / 21}=0.62 ;$ (c) of double antidodal type (not a lifted state) for $\alpha^{12 / 21}=0.05 \pi, \sigma^{12 / 21}=0.28$; (d) of lifted splay type for $\alpha^{12}=0.3 \pi, \alpha^{21}=0.4 \pi, \sigma^{12 / 21}=0.8$, and $\epsilon=0.01$. In the lower panels phase differences between the two layers are indicated by $\Delta a \equiv a_{i}^{1}-a_{i}^{2}$, and between the two new antipodal states (c) by $\psi^{1}, \psi^{2}$.

the other states as well:

$$
\begin{aligned}
& \dot{\delta \phi}_{i}^{\mu}=\frac{1}{N} \sum_{j=1}^{N}\left[\sin \left(\Delta a+\beta^{\mu}\right) \cos \left(\Delta a+\alpha^{\mu \mu}\right) \Delta_{i j}^{\mu \mu} \delta \phi-\right. \\
& \left.\sin \left(\Delta a+\alpha^{\mu \mu}\right) \delta \kappa_{i j}^{\mu}\right]-\sum_{\nu=1}^{M} \sigma^{\mu \nu} \cos \left(\Delta a+\alpha^{\mu \nu}\right) \Delta_{i j}^{\mu \nu} \delta \phi \\
& \dot{\delta \kappa_{i j}^{\mu}}=-\epsilon\left(\delta \kappa_{i j}^{\mu}+\cos \left(\Delta a+\beta^{\mu}\right) \Delta_{i j}^{\mu \mu} \delta \phi\right)
\end{aligned}
$$

where $\Delta_{i j}^{\mu \nu} \delta \phi \equiv \delta \phi_{i}^{\mu}-\delta \phi_{j}^{\nu}$.

In duplex networks, the coupling structure is given by a $2 \times 2$ block matrix $M$ with the $N \times N$ unity matrix $\mathbb{I}_{N}$ :

$$
M=\left(\begin{array}{cc}
A & m \cdot \mathbb{I}_{N} \\
n \cdot \mathbb{I}_{N} & B
\end{array}\right)
$$

If $A$ and $B$ are diagonalizable $N \times N$ matrices which commute $(m, n \in \mathbb{R}, n \neq 0)$, the following relation for the characteristic polynomial can be proven [102] using Schur's decomposition [96, 97]:

$$
\mu^{2}-\left(\left(d_{A}\right)_{i}+\left(d_{B}\right)_{i}\right) \mu+\left(d_{A}\right)_{i}\left(d_{B}\right)_{i}-m n=0
$$

where $\left(d_{A}\right)_{i}$ and $\left(d_{B}\right)_{i}$ are the diagonal elements of the corresponding diagonal matrices of $A$ and $B$, respectively. Note that Eq. (6) not only simplifies the calculation for 


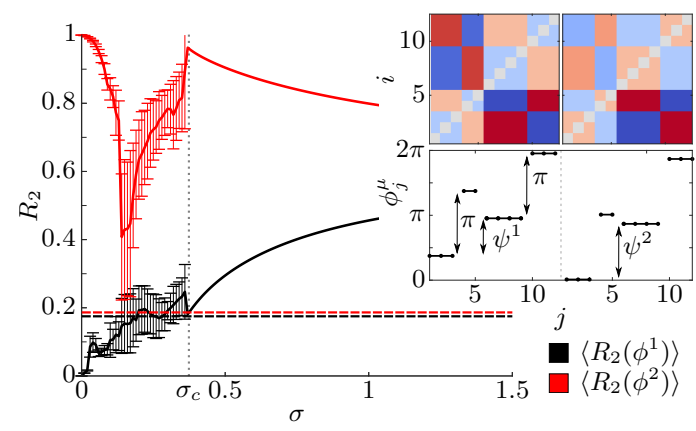

FIG. 3. Birth of double antipodal state in a duplex network $(N=12)$ for a wide range of inter-layer coupling strength $\sigma=\sigma^{12}=\sigma^{21}$. The solid lines are the temporal averages for the second moment order parameter $R_{2}$ of the individual layers (layer 1: black, layer 2: red). The error bars for $\sigma<\sigma_{c}$ denote the standard deviation of the temporal evolution of $R_{2}$. The dashed horizontal lines represent the unique values of $R_{2}$ for the double antipodal state in a monoplex network. The plot was obtained by adiabatic continuation of a duplex double antipodal state (see inset) in both directions starting from $\sigma=0.5$. Parameters: $\alpha^{11 / 22}=0.3 \pi, \alpha^{12 / 21}=0.05$, $\beta^{1}=0.1 \pi, \beta^{2}=-0.95 \pi$, and $\epsilon=0.01$.

the eigenvalues in the case of a duplex structure, moreover, it is a general result on linear dynamical systems on duplex networks. Therefore, this result is important for the investigation of stability and symmetry in multiplex networks.

In the case of a duplex antipodal one-cluster state Eq. (1) with $a_{i}^{1} \in\{0, \pi\}$ and $a_{i}^{2}=a_{i}^{1}-\Delta a$, Eq. (4) can be brought to the form (5) and possesses the following set of Lyapunov exponents $\mathcal{S}=$ $\left\{-\epsilon,\left(\lambda_{i, 1}, \lambda_{i, 2}, \lambda_{i, 3}, \lambda_{i, 4}\right)_{i=1, \ldots, N}\right\}$ where $\lambda_{i, 1, \ldots, 4}$ are the solutions of polynomials containing the eigenvalues of the monoplex system [102].

Thus, the stability analysis of the duplex system is reduced to that of the monoplex case. We are able to analyze the stabilizing and destabilizing features of a duplex network numerically and analytically. To illustrate the effect of multiplexing, the interaction between two clusters of antipodal type is presented in Fig. 4. The stability of these states is determined by integrating Eq. (1) numerically starting with a slightly perturbed lifted antipodal state. The states are stable if the numerical trajectory is approaching the lifted antipodal state. Otherwise, the state is considered as unstable. The black contour lines in Fig. 4 show the borders of the stability regions in dependence of the coupling strength $\sigma^{21}$, as calculated from the Lyapunov exponents. The borders are in remarkable agreement with the numerical results.

In Figure 4, the parameters for the first layer $\alpha^{11}, \beta^{1}$ are chosen such that the antipodal state is stable without inter-layer coupling. The stability of the duplex antipodal states is displayed in the $\left(\alpha^{22}, \beta^{2}\right)$ parameter plane for several values of the inter-layer coupling $\sigma^{21}$ (the stabil-
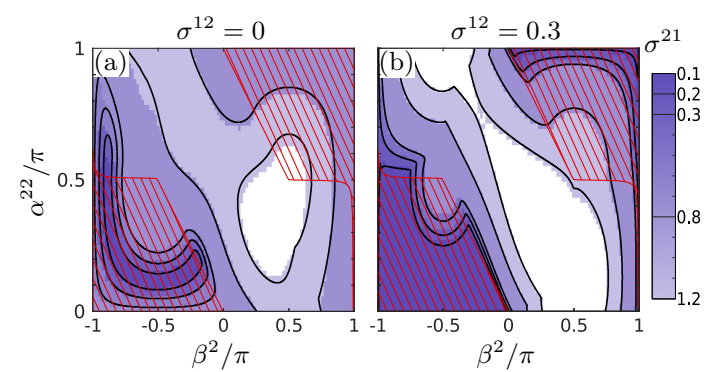

FIG. 4. Regions of stability (blue) and instability (white) of the lifted antipodal state in the $\left(\alpha^{22}, \beta^{2}\right)$ parameter plane for different values of interlayer coupling (indicated by different blue shading) $\sigma^{21}$, where regions of stronger coupling $\sigma^{21}$ (lighter blue) include such of weaker $\sigma^{21}$ (darker blue). Stability regions for single-layer antipodal clusters are indicated by red hatched areas. The inter-layer coupling is considered as (a) unidirectional $\left(\sigma^{12}=0\right)$ and $(\mathrm{b})$ bidirectional $\left(\sigma^{12}=\sigma^{21}\right)$. Parameters: $\alpha^{11}=0.2 \pi, \beta^{1}=-0.8 \pi, \alpha^{12}=0, \alpha^{21}=0.3 \pi$, and $\epsilon=0.01$.

ity regions for smaller values of $\sigma^{21}$ are always contained in regions of larger ones). To compare the effects of the duplex network with the mono-layer case, the stability regions for monoplex antipodals states are displayed as red hatched areas. They are markedly different. In Figure 4(a), the two layers are connected unidirectionally $\left(\sigma^{12}=0\right)$. It can be seen that with increasing inter-layer coupling weight $\sigma^{21}$ the region of stability for the lifted antipodal state also grows. Already for small values of the inter-layer couplings $\sigma^{21}$, a stabilizing effect of the duplex network can be noticed. For $\sigma=0.1$ there exist already regions for which the duplex antipodal state is stable but the corresponding monoplex state would not be stable. The opposite effect is found as well where the duplex network destabilizes a lifted state. Figure 4(b) shows the results for two layers with bidirectional coupling. Here, the duplex structure can have stabilizing and destabilizing effects. Further, for the bidirectional coupling we also notice a growth of the stability region with increasing $\sigma^{21}$ similar to the unidirectional case. However, the regions of stability grow at different rates in dependence on $\sigma^{21}$ and non-monotonically with respect to the parameters $\alpha^{22}, \beta^{2}$. Comparing the size of the stability region for both cases, one can see that for small values of $\sigma^{21}$ the region for bidirectional coupling is larger. In turn, for higher inter-layer coupling, the regions for the unidirectional case are larger.

In conclusion, we have proposed a concept to induce diverse partial synchronization patterns (phase clusters) in adaptively coupled phase oscillator networks. While adaptive networks have recently attracted a lot of attention in the fields of neuro- and social sciences, biology, engineering, and other disciplines, and multilayer networks are a paradigm for real-world complex networks, little has been known about the interplay of multilayer structures and adaptivity. We have aimed to fill this gap 
within a rigorous framework of theoretical analysis and computer simulations. We have shown that multiplexing in a multilayer with symmetry can induce various stable phase cluster states like splay states, antipodal states, and double antipodal states, in a situation where they are not stable or do not even exist in the single layer. Further, we have developed a novel method for analysis of Laplacian matrices of duplex networks which allows for insight into the spectral structure of these networks, and can easily be generalized to more than two layers [102]. This new approach of multiplex decomposition has a broad range of applications to physical, biological, socio-economic, and technological systems, ranging from plasticity in neurodynamics or the dynamics of linear diffusive systems $[98,99]$ to generalizations of the master stability approach $[100,101]$ for adaptive networks [102]. We have used the multiplex decomposition to provide analytic results for the stability of lifted states in the multilayer system. As local dynamics we have used the paradigmatic Kuramoto phase oscillator model, supplemented by adaptivity of the link strengths with a phase lag parameter which can model a whole range of adaptivity rules from Hebbian via spike-timing dependent plasticity to anti-Hebbian.

This work was supported by the German Research Foundation DFG (Projects SCHO 307/15-1 and YA 225/3-1 and Projektnummer - 163436311 - SFB 910). We thank Serhiy Yanchuk for insightful discussions.

* rico.berner@physik.tu-berlin.de

† schoell@physik.tu-berlin.de

[1] H. Markram, J. Lübke, and B. Sakmann, Science 275, 213 (1997).

[2] L. F. Abbott and S. Nelson, Nat. Neurosci. 3, 1178 (2000).

[3] N. Caporale and Y. Dan, Annu. Rev. Neurosci. 31, 25 (2008).

[4] C. Meisel and T. Gross, Phys. Rev. E 80, 061917 (2009).

[5] L. Lücken, O. Popovych, P. Tass, and S. Yanchuk, Phys. Rev. E 93, 032210 (2016).

[6] S. Jain and S. Krishna, Proc. Natl. Acad. Sci. 98, 543 (2001).

[7] T. Gross, C. J. D. D'Lima, and B. Blasius, Phys. Rev. Lett. 96, 208701 (2006).

[8] T. Gross and B. Blasius, J. R. Soc. Interface 5, 259 (2008).

[9] A. Pikovsky, M. G. Rosenblum, and J. Kurths, Synchronization: a universal concept in nonlinear sciences (Cambridge University Press, Cambridge, 2001).

[10] S. H. Strogatz, Nature 410, 268 (2001).

[11] R. Albert and A. L. Barabási, Rev. Mod. Phys. 74, 47 (2002).

[12] M. E. J. Newman, SIAM Review 45, 167 (2003).

[13] S. Boccaletti, A. N. Pisarchik, C. I. del Genio, and A. Amann, Synchronization: From Coupled Systems to Complex Networks (Cambridge University Press, Cam- bridge, 2018).

[14] T. Dahms, J. Lehnert, and E. Schöll, Phys. Rev. E 86, 016202 (2012).

[15] Y. Kuramoto and D. Battogtokh, Nonlin. Phen. in Complex Sys. 5, 380 (2002).

[16] D. M. Abrams and S. H. Strogatz, Phys. Rev. Lett. 93, 174102 (2004).

[17] M. J. Panaggio and D. M. Abrams, Nonlinearity 28, R67 (2015).

[18] T. Aoki and T. Aoyagi, Phys. Rev. Lett. 102, 034101 (2009).

[19] T. Aoki and T. Aoyagi, Phys. Rev. E 84, 066109 (2011).

[20] V. I. Nekorkin and D. V. Kasatkin, AIP Conf. Proc. 1738, 210010 (2016).

[21] D. V. Kasatkin and V. I. Nekorkin, Radiophysics and Quantum Electronics 58, 877 (2016).

[22] R. Berner, E. Schöll, and S. Yanchuk, "Multiclusters in networks of adaptively coupled phase oscillators," SIAM J. Appl. Dyn. Syst. 18, 2227 (2019).

[23] D. V. Kasatkin, S. Yanchuk, E. Schöll, and V. I. Nekorkin, Phys. Rev. E 96, 062211 (2017).

[24] R. Berner, J. Fialkowski, D. Kasatkin, V. Nekorkin, E. Schöll, and S. Yanchuk, "Hierarchical frequency clusters in adaptive networks of phase oscillators," Chaos 29, 103134 (2019).

[25] D. V. Kasatkin and V. I. Nekorkin, Chaos 28, 093115 (2018).

[26] A. Gushchin, E. Mallada, and A. Tang, in Information Theory and Applications Workshop ITA 2015, San Diego, CA, USA (IEEE, 2015) pp. 291-300.

[27] C. B. Picallo and H. Riecke, Phys. Rev. E 83, 036206 (2011).

[28] L. Timms and L. Q. English, Phys. Rev. E 89, 032906 (2014).

[29] Q. Ren and J. Zhao, Phys. Rev. E 76, 016207 (2007).

[30] V. Avalos-Gaytán, J. A. Almendral, I. Leyva, F. Battiston, V. Nicosia, V. Latora, and S. Boccaletti, Phys. Rev. E 97, 042301 (2018).

[31] L. Papadopoulos, J. Z. Kim, J. Kurths, and D. S. Bassett, Chaos An Interdiscip. J. Nonlinear Sci. 27, 073115 (2017).

[32] D. V. Kasatkin and V. I. Nekorkin, Eur. Phys. J. Spec. Top. 227, 1051 (2018).

[33] D. V. Kasatkin, V. V. Klinshov, and V. I. Nekorkin, Phys. Rev. E 99, 1 (2019).

[34] J. Lehnert, P. Hövel, A. A. Selivanov, A. L. Fradkov, and E. Schöll, Phys. Rev. E 90, 042914 (2014).

[35] S. Boccaletti, G. Bianconi, R. Criado, C. I. del Genio, J. Gómez-Gardeñes, M. Romance, I. Sendiña Nadal, Z. Wang, and M. Zanin, Phys. Rep. 544, 1 (2014).

[36] M. De Domenico, A. Solé-Ribalta, E. Cozzo, M. Kivelä, Y. Moreno, M. A. Porter, S. Gómez, and A. Arenas, Phys. Rev. X 3, 041022 (2013).

[37] M. De Domenico, V. Nicosia, A. Arenas, and V. Latora, Nat. Commun. 6, 6864 (2015).

[38] M. Kivelä, A. Arenas, M. Barthélemy, J. P. Gleeson, Y. Moreno, and M. A. Porter, J. Complex Networks 2, 203 (2014).

[39] M. Girvan and M. E. J. Newman, Proc. Natl. Acad. Sci. USA 99, 7821 (2002).

[40] R. Amato, N. E. Kouvaris, M. S. Miguel, and A. DíazGuilera, New J. Phys. 19, 123019 (2017).

[41] R. Amato, A. Díaz-Guilera, and K.-K. Kleineberg, Sci. Rep. 7, 7087 (2017). 
[42] A. Cardillo, M. Zanin, J. Gòmez Gardeñes, M. Romance, A. Garcia del Amo, and S. Boccaletti, Eur. Phys. J. ST 215, 23 (2013).

[43] D. Meunier, R. Lambiotte, and E. T. Bullmore, Front. Neurosci. 4, 200 (2010).

[44] B. Bentley, R. Branicky, C. L. Barnes, Y. L. Chew, E. Yemini, E. T. Bullmore, P. E. Vétes, and W. R. Schafer, PLOS Comput. Biol. 12, 1 (2016).

[45] F. Battiston, V. Nicosia, M. Chavez, and V. Latora, Chaos 27, 047404 (2017).

[46] M. Vaiana and S. F. Muldoon, J. Nonlinear Sci. , 1 (2018).

[47] L. Ramlow, J. Sawicki, A. Zakharova, J. Hlinka, J. C. Claussen, and E. Schöll, EPL 126, 50007 (2019).

[48] A. Ashourvan, Q. K. Telesford, T. Verstynen, J. M. Vettel, and D. S. Bassett, PLoS One 14, e0215520 (2019).

[49] C. Zhou, L. Zemanová, G. Zamora, C. C. Hilgetag, and J. Kurths, Phys. Rev. Lett. 97, 238103 (2006).

[50] C. Zhou, L. Zemanová, G. Zamora-López, C. C. Hilgetag, and J. Kurths, New J. Phys. 9, 178 (2007).

[51] R. Wang, P. Lin, M. Liu, Y. Wu, T. Zhou, and C. Zhou, Phys. Rev. Lett. 123, 38301 (2019).

[52] X. Zhang, S. Boccaletti, S. Guan, and Z. Liu, Phys. Rev. Lett. 114, 038701 (2015).

[53] V. A. Maksimenko, V. V. Makarov, B. K. Bera, D. Ghosh, S. K. Dana, M. V. Goremyko, N. S. Frolov, A. A. Koronovskii, and A. E. Hramov, Phys. Rev. E 94, 052205 (2016).

[54] S. Jalan and A. Singh, Europhys. Lett. 113, 30002 (2016).

[55] S. Ghosh, A. Kumar, A. Zakharova, and S. Jalan, Europhys. Lett. 115, 60005 (2016).

[56] I. Leyva, R. Sevilla-Escoboza, I. Sendiña-Nadal, R. Gutiérrez, J. M. Buldú, and S. Boccaletti, Sci. Rep. 7, 45475 (2017).

[57] R. G. Andrzejak, G. Ruzzene, and I. Malvestio, Chaos 17, 053114 (2017).

[58] S. Ghosh, A. Zakharova, and S. Jalan, Chaos Solitons Fractals 106, 56 (2018).

[59] N. Semenova and A. Zakharova, Chaos 28, 051104 (2018).

[60] M. Mikhaylenko, L. Ramlow, S. Jalan, and A. Zakharova, Chaos 29, 023122 (2019).

[61] J. Sawicki, I. Omelchenko, A. Zakharova, and E. Schöll, Phys. Rev. E 98, 062224 (2018).

[62] I. Omelchenko, T. Hülser, A. Zakharova, and E. Schöll, Front. Appl. Math. Stat. 4, 67 (2019).

[63] E. Rybalova, T. Vadivasova, G. Strelkova, V. Anishchenko, and A. Zakharova, Chaos 29, 033134 (2019).

[64] D. Nikitin, I. Omelchenko, A. Zakharova, M. Avetyan, A. L. Fradkov, and E. Schöll, Phil. Trans. R. Soc. A 377, 20180128 (2019).

[65] K. A. Blaha, K. Huang, F. Della Rossa, L. M. Pecora, M. Hossein-Zadeh, and F. Sorrentino, Phys. Rev. Lett. 122, 014101 (2019).

[66] R. Sevilla-Escoboza, I. Sendiña-Nadal, I. Leyva, R. Gutiérrez, J. M. Buldú, and S. Boccaletti, Chaos An Interdiscip. J. Nonlinear Sci. 26, 065304 (2016).

[67] R. J. Requejo and A. Díaz-Guilera, Phys. Rev. E 94, 022301 (2016).

[68] E. Pitsik, V. Makarov, D. Kirsanov, N. Frolov, M. Goremyko, X. Li, Z. Wang, A. Hramov, and S. Boccaletti, New J. Phys. 20, 075004 (2018).

[69] I. Leyva, I. Sendiña-Nadal, R. Sevilla-Escoboza, V. P.
Vera-Avila, P. Chholak, and S. Boccaletti, Sci. Rep. 8, 8629 (2018).

[70] S. Jalan, A. Kumar, and I. Leyva, Chaos An Interdiscip. J. Nonlinear Sci. 29, 041102 (2019).

[71] N. S. Frolov, V. A. Maksimenko, V. V. Makarov, D. V. Kirsanov, A. E. Hramov, and J. Kurths, Phys. Rev. E 98, 022320 (2018).

[72] O. V. Maslennikov and V. I. Nekorkin, Chaos 28, 121101 (2018).

[73] M. V. Goremyko, D. V. Kirsanov, V. O. Nedaivozov, V. V. Makarov, and A. E. Hramov, in Dyn. Fluctuations Biomed. Photonics XIV, Vol. 10063, edited by V. V. Tuchin, K. V. Larin, M. J. Leahy, and R. K. Wang (2017) p. $100631 \mathrm{C}$.

[74] A. Citri and R. C. Malenka, Neuropsychopharmacology 33, 18 (2008).

[75] E. Edelmann, E. Cepeda-Prado, and V. Leßmann, Front. Synaptic Neurosci. 9, 032805 (2017).

[76] F. Zenke, E. J. Agnes, and W. Gerstner, Nat. Commun. 6, 6922 (2015).

[77] Y. S. Virkar, W. L. Shew, J. G. Restrepo, and E. Ott, Phys. Rev. E 94, 042310 (2016).

[78] R. A. John, F. Liu, N. A. Chien, M. R. Kulkarni, C. Zhu, Q. Fu, A. Basu, Z. Liu, and N. Mathews, Adv. Mater. 30, 1800220 (2018).

[79] T. Gross, C. J. D. D'Lima, and B. Blasius, Phys. Rev. Lett. 96, 208701 (2006).

[80] S. Shai and S. Dobson, Phys. Rev. E 87, 042812 (2013).

[81] L. Wardil and C. Hauert, Sci. Rep. 4, 5725 (2015).

[82] P. Klimek, M. Diakonova, V. M. Eguíluz, M. San Miguel, and S. Thurner, New J. Phys. 18, 083045 (2016).

[83] H. Sakaguchi and Y. Kuramoto, Prog. Theor. Phys 76, 576 (1986).

[84] F. C. Hoppensteadt and E. M. Izhikevich, Biol. Cybern. 75, 129 (1996).

[85] P. Seliger, S. C. Young, and L. S. Tsimring, Phys. Rev. E 65, 041906 (2002).

[86] T. Aoki, Neural Networks 62, 11 (2015).

[87] D. Hebb, The Organization of Behavior: A Neuropsychological Theory, new edition ed. (Wiley, New York, 1949).

[88] Y. Maistrenko, B. Lysyansky, C. Hauptmann, O. Burylko, and P. A. Tass, Phys. Rev. E 75, 066207 (2007).

[89] O. Popovych, S. Yanchuk, and P. Tass, Sci. Rep. 3, 2926 (2013).

[90] Y. Kuramoto, Chemical Oscillations, Waves and Turbulence (Springer-Verlag, Berlin, 1984).

[91] S. H. Strogatz, Physica D 143, 1 (2000).

[92] J. A. Acebrón, L. L. Bonilla, C. J. P. Vicente, F. Ritort, and R. Spigler, Rev. Mod. Phys. 77, 137 (2005).

[93] A. Pikovsky and M. G. Rosenblum, Phys. Rev. Lett. 101, 264103 (2008).

[94] O. E. Omel'chenko and M. Wolfrum, Phys. Rev. Lett. 109, 164101 (2012).

[95] C. U. Choe, T. Dahms, P. Hövel, and E. Schöll, Phys. Rev. E 81, 025205(R) (2010).

[96] S. Boyd and L. Vandenberghe, Convex Optimization (Cambridge University Press, 2004).

[97] J. Liesen and V. Mehrmann, Linear Algebra, Springer Undergraduate Mathematics Series (Springer International Publishing, Cham, 2015).

[98] S. Gómez, A. Díaz-Guilera, J. Gómez-Gardeñes, C. J. 
Pérez Vicente, Y. Moreno, and A. Arenas, Phys. Rev. Lett. 110, 028701 (2013).

[99] A. Solé-Ribalta, M. De Domenico, N. E. Kouvaris, A. Díaz-Guilera, S. Gómez, and A. Arenas, Phys. Rev. E 88, 032807 (2013).

[100] L. M. Pecora and T. L. Carroll, Phys. Rev. Lett. 80, 2109 (1998)

[101] L. Tang, X. Wu, J. Lü, J. Lu, and R. M. D’Souza, Phys.
Rev. E 99 (2019), 012304.

[102] See Supplemental Material at http... for phase differences of duplex one-cluster states, the characteristic polynomial of duplex matrices, a general form of the multiplex decomposition, a derivation of the Lyapunov exponents of duplex antipodal states, and for a numerical analysis of the influence of inhomogeneous natural frequencies on the emergence of duplex double antipodal clusters. 